\title{
ELECTRON SELF-TRAPPING IN A FLUID CAPABLE OF A MEAN-FIELD PHASE TRANSITION
}

\author{
J.P. HERNANDEZ and L.W. MARTIN \\ Department of Physics and Astronomy, University of North Carolina, Chapel Hill, NC 27599-3255, \\ U.S.A.
}

\begin{abstract}
The conditions necessary for self-trapping of a quantum particle in a fluid are reviewed, using a mean-field model. A thermodynamic approach is used along with density functional methods. The possibility that self-trapping may nucleate a heterophase region in the fluid is discussed, emphasizing the equilibrium requirement of a pressure difference across a curved surface between coexisting phases. Numerical results for an excess electron in fluid neon are summarized.
\end{abstract}

It is known that an electron may self-trap in a fluid by distorting the local fluid structure. In this work, a description of the most probable state of the electron-fluid system is discussed. A thermodynamic approach, using a free energy model and density functional methods, is reviewed. The approach includes the possibility that self-trapping may nucleate a local heterophase region in the fluid. The electron-fluid interaction is described by energy-dependent atomic pseudopotentials arising from electron-atom interactions which are assumed to be non-overlapping. The fluid itself is described via local equations of state. The change in the most probable state from a delocalized electron to a selftrapped one has experimentally observable consequences.

The formalism ${ }^{1}$ used to discuss the self-trapping problem will be reviewed first. The possibility ${ }^{2}$ of nucleation of a local heterophase region will be included in the formalism. Applications to excess electrons in fluid neon will be summarized with full results to be presented elsewhere ${ }^{3}$.

A thermodynamic approach to this problem allows substantial simplification. It uses the fluid variables of temperature $(T)$, density $(\rho)$, pressure $(P)$, and chemical potential $(\mu)$. For temperatures below the critical one $\left(T_{c}\right)$, for the vapor-liquid phase transition, the surface tension $(\gamma)$ and the concept of a "surface of coexistence" are needed. The latter concept is one which treats an interfacial region (i.e. one in which the fluid density takes on values between that of the vapor $\left(\rho_{\mathrm{V}}\right)$ and the liquid $\left(\rho_{\mathrm{L}}\right)$ at coexistence) by ignoring its detailed structure and assuming $\rho=\rho_{V}$ or $\rho_{L}$ with a jump at the "equimolecular dividing surface" - the one for which the piecewise constant density approximation 
conserves the number of atoms in the system. ${ }^{4}$ To treat a system consisting of an electron and the fluid, a reference system is chosen: a homogeneous fluid at $\rho$ and $T$ with the electron in its lowest energy delocalized state. In this reference system, the electron-fluid interaction is characterized by a uniform potential $\left(\mathrm{V}_{\rho}\right)$ which depends on the fluid density. A density functional model is then sought to describe the most probable state of the $(\rho, T)$ fluid-electron system. Such a state is described by an electron wave function $\psi(r)$ and an inhomogeneous fluid density $\rho(r)$. If this density is slowly varying, except in a possible interfacial region, local values for $\mu, \mathrm{P}$ and $\mathrm{V}$ may be assumed.

The state sought is specified by the Schröedinger equation for the electron,

$$
\left.\left[-\frac{h^{2}}{2 m} \nabla^{2}+\left(V(r)-V_{\rho}\right)\right)-E\right] \psi(r)=0,
$$

and by the Helmholtz free energy difference between this system and the reference one,

$$
\Delta F=E+\int\left[\rho(r)\left(\mu(r)-\mu_{\rho}\right)-\left(P(r)-P_{\rho}\right)\right] d r+\gamma \int d A
$$

The subscripted variables refer to the reference system and the local ones to the system sought. The last term in (2) is the surface energy due to a possible heterophase region; for simplicity, $\gamma$ is assumed independent of surface curvature. As it is reasonable to assume spherical symmetry, then $\int \mathrm{d} A=4 \pi \mathrm{R}^{2}$ with $R$ being the radius of the equimolecular dividing surface. For the most probable system state, (2) must be a minimum; thus it is to be extremized with respect to $\rho(r)$ and $R$.

For all $\mathrm{r}$ except within the interfacial region $\left(\rho_{V}<\rho(r)<\rho_{L}\right)$, if any, $\delta \Delta F / \delta \rho(r)=0$ implies

$$
\left[\rho(r) \frac{\delta \mu(r)}{\delta \rho(r)}-\frac{\delta P(r)}{\delta \rho(r)}\right]+\left\{(\mu(r)-\mu \rho)+\frac{\delta V(r)}{\delta \rho(r)} \frac{|\psi(r)|^{2}}{\langle\psi \mid \psi\rangle}\right\}=0
$$

$\langle\psi \mid \psi\rangle$ is the normalization. The local relation among density, chemical potential, and pressure guarantees that the square bracket in (3) vanishes. Thus, the requirement that the curly bracket vanish yields an implicit relation between $\rho(r)$ and $\psi(r)$ which is to be used to find a self-consistent $V(r)$ for (1). That equation may then be integrated numerically to find its ground state. If there is an interfacial region, the approximation $\rho(r)=\rho_{V}$ or $\rho_{L}$ with a jump at $R$ defines $V(r)$ within that region. Note that for $V(r) \alpha \rho(r)$, continuity of $\psi(r)$ implies that the interfacial region is a surface, not a volume, and $R$ is uniquely specified. This uniqueness is not a general one and ignorance of $\rho(r)$ in the interfacial region will require a method for defining, or at least bounding, $R$.

Extremization of (2) with $\mathrm{R}$ is to be accomplished at constant $\mu, \mathrm{P}, \mathrm{T}$, total number of atoms, and total volume. 5 Thus, it yields a pressure difference across $\mathrm{R}$ : 


$$
-\left[P\left(R^{-}\right)-P\left(R^{+}\right)\right]+\left[V\left(R^{-}\right)-V\left(R^{+}\right)\right] \frac{|\psi(R)|^{2}}{\langle\psi \mid \psi\rangle}+\frac{2 \gamma}{R}=0
$$

The inner region is the heterophase one, and it should be recalled through the interfacial region $\mu(r)=\mu\left(\rho_{V}\right)=\mu\left(\rho_{L}\right)$. Defining $r_{V, L}$ as the radii at which (3) yields the coexistence densities $\left(\rho_{V, L}\right)$ brackets $\mathbf{R}$. This bracketing allows limiting calculations at the extreme bounds for $\mathbf{R}$.

Equation (2) may now be artifically separated into a "surface" term,

$$
\Delta \mathrm{Fl}_{\mathrm{S}}=\frac{4 \pi}{3} \mathrm{R}^{3}\left\{\left[\mathrm{~V}\left(\mathrm{R}^{+}\right)-\mathrm{V}\left(\mathrm{R}^{-}\right)\right] \frac{|\psi(\mathrm{R})|^{2}}{\langle\psi \mid \psi\rangle}+\frac{\gamma}{\mathrm{R}}\right\},
$$

from the surface pressure terms in (4) (which are constant except for the jump at R) and the last term in (2), and the remainder of (2).

The nucleation of a heterophase region has the necessary conditions: $T<T_{c}$ and $\rho>\rho_{L}$ if $V(r)>0$, or $\rho<\rho V$ if $V(r)<0$. A value of $\gamma$ is needed, but only to obtain a result from (5). A reasonable expression, scaled with the law of corresponding states, is 6

$$
\gamma=\left(\mathrm{k}_{\mathrm{B}} \mathrm{T}_{\mathrm{c}} \rho_{\mathrm{c}}^{2 / 3}\right) \times 2\left(1-\mathrm{T} / \mathrm{T}_{\mathrm{c}}\right)
$$

Numerical results can be obtained with this formalism, given an equation of state $(P(\rho)$ and $\mu(\rho)$ and specifying $V(\rho)$ ). We have used the van der Waals relations and, for low energy electrons and atoms with a positive scattering length, if overlaps are neglected, an s-wave pseudopotential.

$$
V(r)=-\frac{h^{2}}{2 m} 4 \pi \frac{\tan \delta_{0}(k)}{k} \rho(r)
$$

where $\delta_{0}(\mathrm{k})$ are the energy-dependent s-wave scattering phase shifts. The scattering length is defined as the limit as $k \rightarrow 0$ of $\left(-\delta_{0}(k) / k\right)$. The scattering energy $\left(k^{2} k^{2} / 2 m\right)$ is related to fluid density using the Wigner-Seitz model. For slowly varying $\rho(r)$, this model requires a boundary condition from translational invariance:

$$
\tan \left(\mathrm{kr}_{\mathrm{s}}+\delta_{\mathrm{o}}(\mathrm{k})\right)=\mathrm{kr}_{\mathrm{s}}
$$

$\rho(r)^{-1}=4 \pi r_{s}^{3} / 3$. The formalism is now complete.

In carrying out calculations for electrons in fluid neon, the equation of state has been parametrized to the critical data: $\rho_{c}=1.443 \times 10^{22} \mathrm{~cm}^{-3}, T_{c}=44.38 \mathrm{~K}$; the scattering phase shifts were those of O'Malley and Crompton. ${ }^{7}$ We have reported ${ }^{1}$ an analysis suitable to discuss the electron mobility data in 
neon obtained by Borghesani and Santini ${ }^{8}$ for $\mathrm{T}=46.5 \mathrm{~K}$ and $\rho<1.70 \times 10^{22} \mathrm{~cm}^{-3}$. Also, a brief discussion of the heterophase nucleation for $0.95 \geq T / T_{C} \geq 0.80$ and $\rho>\rho_{L}$ has been submitted. ${ }^{2}$ We shall now briefly summarize results yielding a self-trapped state as the most probable one $(\Delta \mathrm{F}<0)$.

In our model for excess electrons in neon, the self-trapped state is stable in a region of $(p, T)$ space. At low densities and temperatures, this region is bounded by the coexistence curve on the vapor side and $\rho \approx 0.75 \rho_{\mathrm{c}}$. As the temperature is raised the minimum density for stable self-trapping rises to approximately $1.4 \rho_{\mathfrak{c}}$ at $1.22 \mathrm{~T}_{\mathrm{c}}$. There is no stable self-trapping at higher temperatures. There is also a maximum density for self-trapping, since the stiffness of the fluid vs distortions increases with density on isotherms. This high density boundary goes from the previously noted value of $1.4 \rho_{c}$ at $1.22 T_{c}$ to approximately $2 p_{c}$ at $T_{c}$. On further lowering of the temperature, stable self-trapping is accompanied by nucleation of a vapor bubble in the liquid. The maximum density for stable self-trapping appears to decrease slightly as $T$ goes from $T_{\mathcal{C}}$ to $0.95 T_{C}$, but this result depends on the value of $\gamma$ chosen. Then, on further temperature decrease, the maximum density increases again, approaching the density of the liquid at coexistence at lower temperatures.

It is noteworthy that the size of the self-trapping region, characterized by the electron's classical turning point in (1), increases on approaching the coexistence curve on the liquid side for $T<T_{c}$. The contribution from (5) to $\Delta \mathrm{F}$, being positive, tends to destabilize self-trapping; the quantum pressure contribution dominates the $\gamma / \mathrm{R}$ term unless the coexistence curve is closely approached. Fractional uncertainties in the values for $\mathrm{R}$ are of order $10 \%$ and those of $\Delta \mathrm{F}$ of less than $20 \%$, as obtained by the bracketing method described after eq. (4). The nucleated vapor bubbles have radii of order $10 \AA$.

The predicted onset of stable self-trapping at $T \sim 1.05 \mathrm{~T}_{\mathrm{c}}$ is in good agreement with mobility measurements. ${ }^{1,8}$ It would be of interest to probe the destabilization of self-trapping due to increasing the temperature for $0.8<\rho / \rho_{c}<1.9$ and to increasing the density for $1<\mathrm{T} / \mathrm{T}_{\mathfrak{c}}<1.2$. Probing densities above $\rho_{\mathrm{L}}$ for $\mathrm{T} / \mathrm{T}_{\mathrm{c}}<1$ would likewise be a useful test of the theoretical predictions.

In summary, a full spectrum of the conditions required for stable self-trapping of a quantum particle in a simple fluid have been described. The possibility that self-trapping nucleates a heterophase region in the fluid has been investigated, including the surface pressure required for equilibrium when a curved surface separates coexisting phases; the surface tension and quantum particle pressure contribute to this equilibrium. Numerical calculations have been carried out for excess electrons in fluid neon. The results are in good agreement with available mobility measurements and experiments are sought to probe other features of these theoretical predictions.

We wish to acknowledge support for this project from our department and the College of Arts and Sciences, UNC-Chapel Hill. 


\section{References}

/1/ HERNANDEZ, J. P., and L. W. MARTIN, Phys. Rev. A 43 (1991) 4568

2/ HERNANDEZ, J. P., and L. W. MARTIN, submitted for publication

13/ MARTIN, L. W., Ph.D. thesis (University of North Carolina, Chapel Hill, 1991) and MARTIN, L. W., and J. P. HERNANDEZ, to be submitted for publication

14/ Molecular Theory of Gases and Liquids by J. O. HIRSCHFELDER, C. F. CURTISS, and R. B. BIRD (John Wiley and Sons, New York, 1954) p. 339

15/ Statistical Physics, Part I by L. D. LANDAU and E. M. LIFSHITZ, 3rd edition (Pergamon, Oxford, 1980) p. 552

16/ JOHNSON, V. J., WADD Technical Report 60-56, Part I (U.S. Department of Commerce, NBS, 1960)

n/ O'MALLEY, T. F., and R. W. CROMPTON, J. Phys. B 23 (1980) 3451

18/ BORGHESANI, A. F., and M. SANTINI Phys. Rev. A 42 (1990) 7377 